\title{
Brownian rectifiers in the presence of temporally asymmetric unbiased forces
}

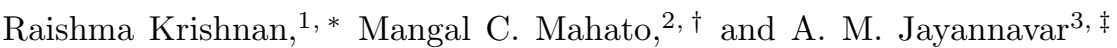 \\ ${ }^{1}$ Institute of Physics, Sachivalaya Marg, Bhubaneswar-751005, India \\ ${ }^{2}$ Department of Physics, North-Eastern Hill University, Shillong-793022, India \\ ${ }^{3}$ Institute of Physics, Sachivalaya Marg, Bhubaneswar 751005, India
}

\begin{abstract}
The efficiency of energy transduction in a temporally asymmetric rocked ratchet is studied. Time asymmetry favours current in one direction and suppresses it in the opposite direction due to which large efficiency $\sim 50 \%$ is readily obtained. The spatial asymmetry in the potential together with system inhomogeneity may help in further enhancing the efficiency. Fine tuning of system parameters considered leads to multiple current reversals even in the adiabatic regime.
\end{abstract}

PACS numbers: 05.40.-a, 05.60.cd, 02.50.Ey.

\section{INTRODUCTION}

Brownian rectifiers or ratchets are devices that convert nonequilibrium fluctuations into useful work in the presence of load. Several physical models [1, 2, 3, 4] have been proposed to understand the nature of currents and their possible reversals with applications in nanoparticle separation devices 4]. The possibility of enhancement of efficiency with which these Brownian rectifiers convert the nonequilibrium fluctuations into useful work has generated much interest in this field. This, in turn, has led to the emergence of a separate subfield - stochastic energetics - on its own right [5, 6]. Using this formalism one can readily establish the compatibility between Langevin or Fokker-Planck formalism with the laws of thermodymanics thereby providing a tool to study systems far from equilibrium. With this framework one can calculate various physical quantities such as efficiency of energy transduction 7], energy dissipation (hysteresis loss), entropy (entropy production) 8], etc.

Most of the studies yield low efficiencies, in the subpercentage range, in various types of ratchets. This is due to intrinsic irreversibility associated with ratchet operation. Only fine tuning of parameters can lead to a large efficiency, the regime of parameters, however, being very narrow [9]. Recently Makhnovskii et al. [10] constructed a special type of flashing ratchet with two asymmetric double-well periodic-potential- states displaced by half a period. Such flashing ratchet models were found to be highly efficient with efficiency an order of magnitude higher than in earlier models [5, 6, 11]. The basic idea behind this enhanced efficiency is that even for diffusive Brownian motion the choice of appropriate potential profile ensures suppression of backward motion and hence reduction in the accompanying dissipation.

In the present work, we study the motion of a particle in a rocking ratchet rocked purposefully as to favour current in one direction but to suppress motion in the opposite direction. This is in similar spirit as in case of flashing ratchets proposed by Makhnovskii et al. 10]. This is accomplished by applying temporally asymmetric but unbiased periodic forcings 12, 13, 14]. Interestingly, such choice of forcings helps in obtaining rectified currents with high efficiency even for spatially symmetric periodic potentials. Still higher efficiency is obtained with asymmetric potentials. The range of parameters of operation of such ratchets is quite wide sustaining large loads. In addition, frictional inhomogeneity may further enhance the efficiency. We also see multiple current reversals in the full parameter space of operation even in the adiabatic regime. However, multiple current reversals require fine tuning of the parameters.

\section{THE MODEL}

The Brownian motion of a particle in an inhomogeneous medium is described by the Langevin equation [15]

$$
\dot{q}=-\frac{V^{\prime}(q)-F(t)}{\gamma(q)}-\frac{k_{B} T \gamma^{\prime}(q)}{2[\gamma(q)]^{2}}+\sqrt{\frac{k_{B} T}{\gamma(q)}} \xi(t)
$$

where $\xi(t)$ is a randomly fluctuating Gaussian thermal noise with zero mean and correlation, $\left\langle\xi(t) \xi\left(t^{\prime}\right)>=\right.$ $2 \delta\left(t-t^{\prime}\right)$. The periodic potential $V(q)=-\sin (q)-$ $(\mu / 4) \sin (2 q)$. The parameter $\mu(-1<\mu<1)$, characterises the degree of asymmetry in the potential. The friction coefficient $\gamma(q)=\gamma_{0}(1-\lambda \sin (q+\phi))$, with $0 \leq \lambda<1$ where $\phi$ is the phase difference. $F(t)$ is the externally applied periodic driving force. The corresponding Fokker-Planck equation [16] is given by

$$
\begin{aligned}
\frac{\partial P(q, t)}{\partial t} & =\frac{\partial}{\partial q} \frac{1}{\gamma(q)}\left[k_{B} T \frac{\partial P(q, t)}{\partial q}\right. \\
& \left.+\left[V^{\prime}(q)-F(t)\right] P(q, t)\right] .
\end{aligned}
$$

Since we are interested in the adiabatic limit we first obtain an expression for the probability current density \begin{tabular}{l|l|} 
*Electronic address: & raishma@iopb.res.in \\
\hline †Electronic address: & mangal@nehu.ac.in \\
‡Electronic address: & jayan@iopb.res.in
\end{tabular} 
$j$ in the presence of a constant external force $F_{0}$. The expression is given by

$$
j=\frac{1-\exp \left[\frac{-2 \pi F_{0}}{k_{B} T}\right]}{\int_{0}^{2 \pi} d y I_{-}(y)},
$$

where $I_{-}(y)$ is given by

$$
\begin{array}{r}
I_{-}(y)=\exp \left[\frac{-V(y)+F_{0} y}{k_{B} T}\right] \\
\int_{y}^{y+2 \pi} d x \quad \gamma(x) \exp \left[\frac{V(x)-F_{0} x}{k_{B} T}\right] .
\end{array}
$$

It may be noted that for $\mu=0, j\left(F_{0}\right) \neq-j\left(-F_{0}\right)$ for $\phi \neq 0, \pi$. This asymmetry ensures rectification of current for the rocked ratchet even in the presence of spatially symmetric potential. We assume that $F(t)$ changes slow enough, i.e., its frequency is smaller than any other frequency related to the relaxation rate in the problem such that the system is in a steady state at each instant of time.

We consider time asymmetric ratchets with a zero mean periodic driving force 12 given by

$$
\begin{aligned}
F(t) & =\frac{1+\epsilon}{1-\epsilon} F_{0}, \quad\left(n \tau \leq t<n \tau+\frac{1}{2} \tau(1-\epsilon)\right), \\
& =-F_{0},\left(n \tau+\frac{1}{2} \tau(1-\epsilon)<t \leq(n+1) \tau\right) .
\end{aligned}
$$

Here, the parameter $\epsilon$ signifies the temporal asymmetry in the periodic forcing. For this forcing in the adiabatic limit the time averaged current is given by 7,12 . $<j>=\frac{1}{2}[j(F(t))+j(-F(t))]$ which is equal to

$$
<j>=\frac{1}{2}\left(j_{1}+j_{2}\right)
$$

with

$$
\begin{aligned}
& j_{1}=(1-\epsilon) j\left(\frac{1+\epsilon}{1-\epsilon} F_{0}\right), \\
& j_{2}=(1+\epsilon) j\left(-F_{0}\right) .
\end{aligned}
$$

The input energy $E_{\text {in }}$ per unit time is given by 7 ]

$$
E_{i n}=\frac{1}{2} F_{0}\left[\left(\frac{1+\epsilon}{1-\epsilon}\right) j_{1}-j_{2}\right]
$$

To calculate efficiency a load $L$ is applied against the direction of current with overall potential given by $V(q)=$ $-\sin (q)-(\mu / 4) \sin (2 q)+q L$. The current flows against the load as long as the load is less than the stopping force $L_{s}$ beyond which the current is in the same direction as that of the load. Thus in the operating range of the load $0<L<L_{s}$ the Brownian particles move in the direction opposite to the load thereby storing energy. The average work done over a period is given by

$$
E_{\text {out }}=\frac{1}{2} L\left[j_{1}+j_{2}\right]
$$

The thermodynamic efficiency of energy transduction is [5, 6] $\eta=E_{\text {out }} / E_{\text {in }}$. In our present discussion all the physical quantities are taken in dimensionless units. In the following section we discuss the results of our calculation. In order to evaluate currents we use the method of Gaussian quadrature.

\section{RESULTS AND DISCUSSIONS}

To begin with we consider a homogeneous system in the presence of spatially symmetric potential. In Fig. 1

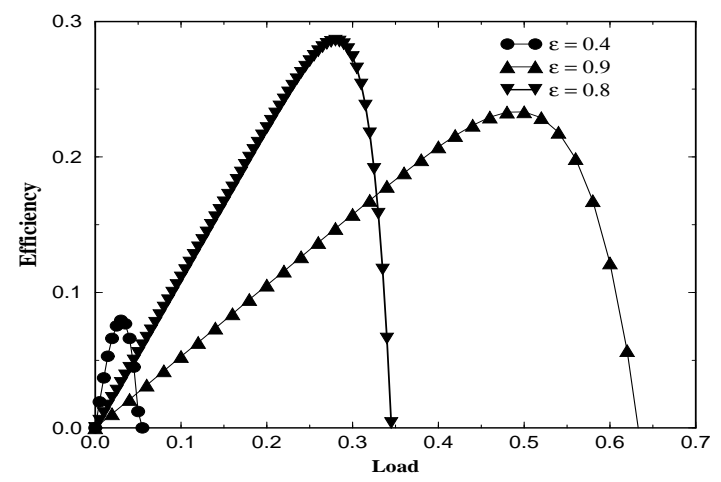

FIG. 1: Efficiency vs load for various values of $\epsilon$ with fixed $F_{0}=0.1$.

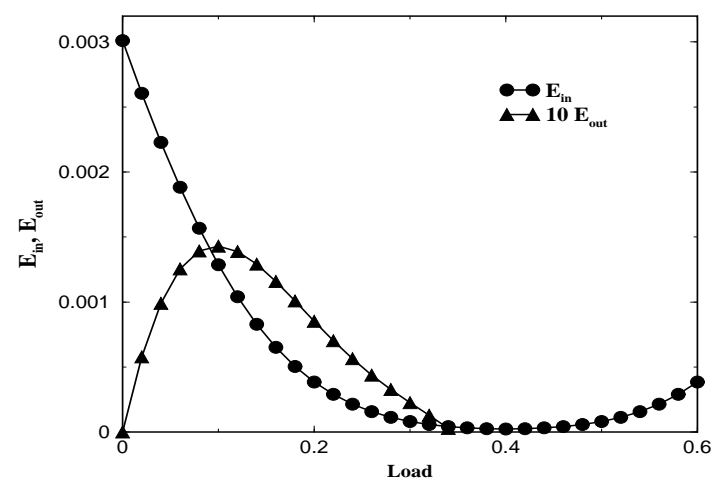

FIG. 2: Input and output energy vs load for $\epsilon=0.8$ with fixed $F_{0}=0.1$. The negative values of the output energy are not shown. The output curve is blown up ten times to scale with the input curve values.

we plot efficiency as a function of load in the presence of temporal asymmetry $\epsilon$ for $F_{0}=0.1$ and $T=0.1$. For a given $\epsilon$ the efficiency slowly increases with load, attains a maximum and then decreases rapidly. The locus of peak values corresponding to different $\epsilon$ values (with appropriate load $L$ ) is found to have a nonmonotonous behaviour with the maximum $(\sim 0.29)$ being at around $\epsilon=0.8$ corresponding to a load of $L=0.258$. For this value of $\epsilon$ there is a large external driving force 


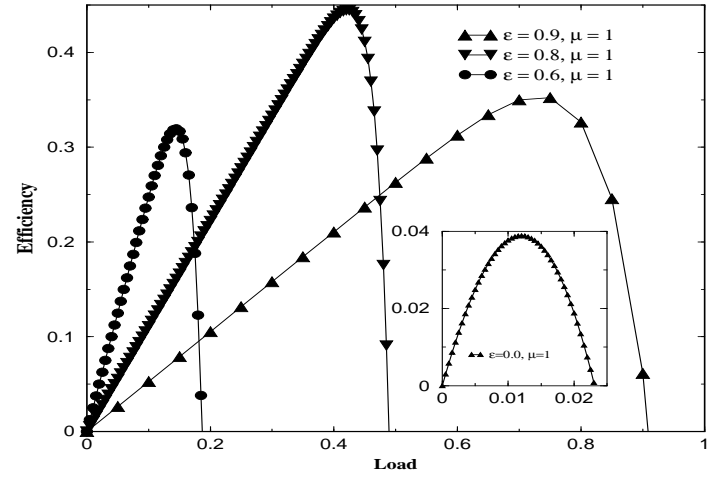

FIG. 3: Efficiency vs load for various $\epsilon$ with fixed $F_{0}=0.1$. Inset shows the efficiency vs load for the case $\epsilon=0$ and $\mu=1$.

field, i.e., $\left(\frac{1+\epsilon}{1-\epsilon}\right) F_{0}\left(=9 F_{0}=0.9\right)$ for a time duration $\tau(1-\epsilon) / 2(=0.1 \tau)$, in one direction which causes considerable reduction in the barrier height in that direction. This makes the particle cross the potential barrier easily and slide down the slope before the field changes. During the other part of the period of $F(t)\left(=-F_{0}=-0.1\right)$ which lasts for $0.9 \tau$ the potential barrier for reverse motion remains large thus nullifying the chances of reverse motion. There is an optimum value of $\epsilon(\sim 0.8$, for the given parameters), however, for which considerable current is obtained. Beyond this value of $\epsilon$ the duration for which the force remains large is so small that eventhough the barriers may be negligible or vanishing altogether, the particle may not have enough opportunity to move away before the force gets changed. This is the reason why the rectifier works most efficiently for an optimum value of $\epsilon$. For finite $\epsilon$ current against the load is obtained for load $L<L_{s} . L_{s}$ represents the range of load for which useful work is performed and is an increasing function of $\epsilon$.

The useful work so obtained $E_{\text {out }}$ and the input energy $E_{\text {in }}$ are shown in Fig. 2 for a representative value of $\epsilon$

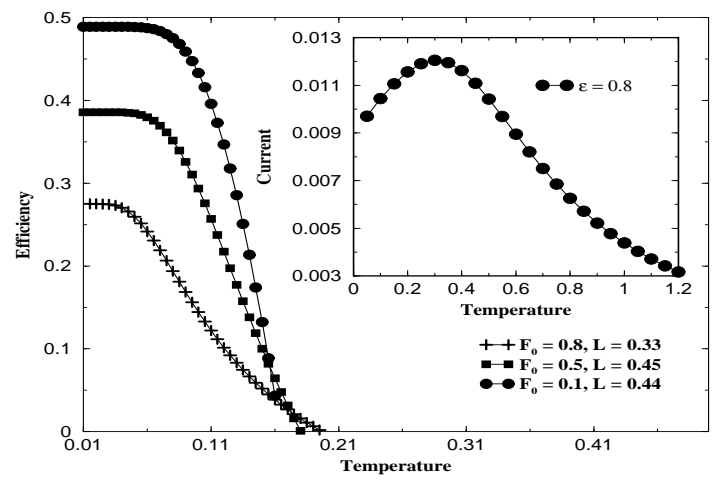

FIG. 4: Efficiency vs temperature with $\mu=1$ for (i) $\epsilon=$ $0.2, F_{0}=0.8$, (ii) $\epsilon=0.4, F_{0}=0.5$ and (iii) $\epsilon=0.8, F_{0}=$ 0.1 . Inset shows current as a function of temperature for $\epsilon=0.8, F_{0}=0.1$ in the absence of load.

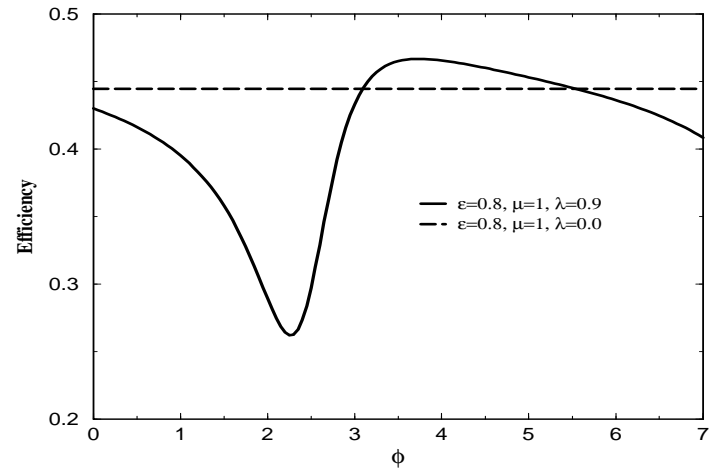

FIG. 5: Efficiency vs $\phi$ for $\epsilon=0.8, \mu=1, L=0.44$ for (i) $\lambda=0.0$ and (ii) $\lambda=0.9$ with fixed $F_{0}=0.1$ and $T=0.1$

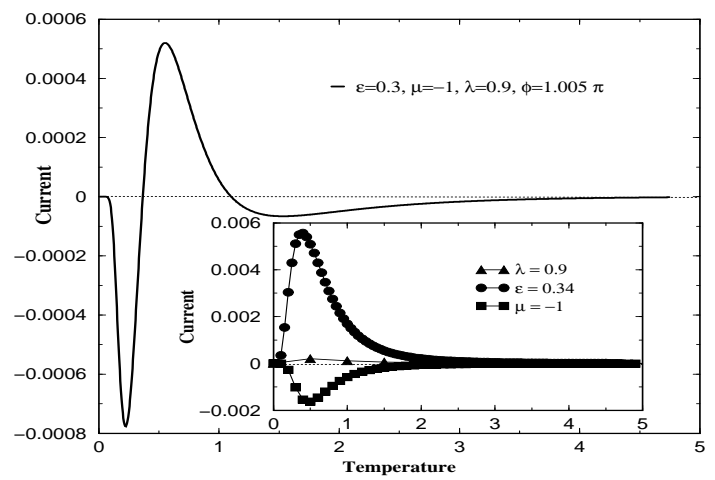

FIG. 6: Current vs temperature curve showing two current reversals for $\mu=-1, \lambda=0.9$ and $\epsilon=0.34$ with $\phi=$ $1.005 \pi, F_{0}=0.3$ and $L=0$. Inset shows the current in the presence of lone asymmetry parameters $(\lambda, \epsilon, \mu)$.

as a function of load. The input energy decreases to a minimum value for a load larger than $L_{s}$. Moreover, it remains positive, as expected, over the entire range. The output energy shows a peak with load in the region where the input energy is monotonously decreasing. It then becomes negative for $L>L_{s}$ as anticipated. The qualitative behaviours of efficiency and energies shown in Figs. 11 and 2] are similar to those in reference [10] for the flashing ratchet.

In Fig. 3 we consider the periodic potential $V(q)$ to be spatially asymmetric together with a temporally asymmetric external driving force field. The potential asymmetry enhances the efficiency of energy transduction as well as widens the range of load. This is due to the fact that for $\epsilon \neq 0$ the presence of asymmetric parameter $\mu(>0)$ further reduces the potential barrier for forward motion and enhances the barrier for backward motion. Moreover, as can be seen from the inset, one can get finite current even when $\epsilon=0$ with finite stopping force $L_{s}$ in contrast to the symmetric potential case. From Figs. [1 and 3 it is clear that the temporally asymmetric forces not only enhance the efficiency of energy transduction 
but also widen the operation range of load against which the ratchet system works.

In Fig. 4 we plot efficiency as a function of $T$ for various $\epsilon$ values in the presence of potential asymmetry $(\mu>0)$. The efficiency decreases with temperature. The relevant physical parameters chosen for optimal efficiency are mentioned in the caption. From the inset it is to be noted that the current peaks as a function of temperature yet efficiency decreases monotonically. This implies that thermal fluctuation do not favour energy transduction in this case. It is worth mentioning that for given temperature and $\epsilon$ the efficiency shows peaking behaviour as a function of $F_{0}$; the efficiency being zero for $F_{0}=0$ as well as for large $F_{0}$ for in these limits output current vanishes in the absence of load.

Next, we present the effect of frictional inhomogeneity $(\gamma=\gamma(q) ; \lambda \neq 0)$. In Fig. 5 we plot the efficiency as a function of the phase difference between the potential and the friction coefficient $\gamma(q)$ for a typical case. We observe that the inclusion of this parameter $\lambda$ further increases the efficiency in a range of $\phi$ depending on other parameter values. It is worth mentioning that for inhomogeneous systems the efficiency peaks with temperature in a limited range of parameters. With frictional inhomogeneity the range of temperature in which one can obtain output current with finite efficiency is extended to a large temperature where contribution of $\lambda$ dominates over other parameters. In Fig $[$ we show that by properly choosing the parameters we can obtain multiple current reversals as a function of temperature. It should be noted that such reversals are not possible in the homogeneous case in the adiabatic regime 17. The inset shows current as a function of individual parameters $(\epsilon, \mu, \lambda)$. The plots indicate that individual parameters cannot bring about current reversals separately. However, the possibility of current reversals arises due to the combined effect of the three asymmetry parameters considered. We have also observed more number of current reversals than shown in Fig. [6 by fine tuning the parameters.

\section{CONCLUSIONS}

We find large efficiency for rocking ratchets driven by temporally asymmetric periodic field the origin of which can be traced to the suppression of backward motion. The observed efficiency is much higher than the earlier reported values eventhough the ratchet operates in an intrinsically irreversible domain. This asymmetry factor has also helped in increasing the range of load of operation of the ratchet. We also observe multiple current reversals in the adiabatic limit by proper fine tuning of different parameters. These reversals are attributed to inherent complex dynamics of the system.

\section{ACKNOWLEDGEMENTS}

AMJ thanks D.-Y. Yang for providing the reference [10] prior to publication. MCM thanks Institute of Physics, Bhubaneswar for hospitality where the present work was carried out.
[1] F. Jülicher, A. Adjari and J. Prost, Rev. Mod. Phys. 69, 1269 (1997).

[2] P. Reimann, Phys. Rep. 361, 57 (2002) and references therein.

[3] A. M. Jayannavar, cond-mat 0107079 in Frontiers in Condensed Matter Physics, (A commemorative volume to mark the $75^{t h}$ year of Indian Journal of Physics), ed. J. K. Bhattacharjee and B. K. Chakrabarti (in press).

[4] Special issue on "Ratchets and Brownian motors: basics, experiments and applications" ed. H. Linke, Appl. Phys. A75(2) 2002.

[5] K. Sekimoto, J. Phys. Soc. Jpn. 66, 6335 (1997).

[6] J. M. R. Parrondo and B. J. De Cisneros, Appl. Phys. A75, 179 (2002).

[7] H. Kamegawa, T. Hondou and F. Takagi, Phys. Rev. Lett. 80, 5251 (1998); F. Takagi and T. Hondou, Phys. Rev. E60, 4954 (1999); D. Dan and A. M. Jayannavar, Phys. Rev. E66, 41106 (2002).

[8] Raishma Krishnan and A. M. Jayannavar, cond-mat 0310726 Debasis Dan and A. M. Jayannavar, cond-mat 0303417

[9] I. M. Sokolov, cond-mat 0207685 1.
[10] Yu.A. Makhnovskii, V. M. Rozenbaum, D.-Y. Yang, S. H. Lin and Tsong, to appear in Phys. Rev. E.

[11] R. D. Astumian, J. Phys. Chem. 100, 19075 (1999).

[12] D. R. Chialvo, M. M. Millonas, Phys. Lett. A 209, 26 (1995).

[13] M. C. Mahato and A. M. Jayannavar, Phys. Lett. A 209, 21 (1995).

[14] Bao-Quan Ai, X. J. Wang, G. T. Liu, H. Z. Xie, D. H. Wen, W. Chen and L. G. Liu, Phys. Rev. E68, 061105 (2003).

[15] A. M. Jayannavar and M. C. Mahato, Pramana-J. Phys. 45, 369 (1995); M. C. Mahato, T. P. Pareek and A. M. Jayannavar, Int. J. Mod. Phys. B 10, 3857 (1996), D. Dan, M. C. Mahato and A. M. Jayannavar, Phys. Lett. A 258, 217 (1999); Int. J. Mod. Phys. B 14, 1585 (2000); Phys. Rev. E60, 6421 (1999); Phys. Rev. E63, 56307 (2001).

[16] H. Risken, The Fokker-Planck Equation (Springer Verlag, Berlin, 1984).

[17] D. Dan, M. C. Mahato and A. M. Jayannavar, Phys. Rev. E63, 056307 (2001). 